\title{
Desempeño de las enfermeras en Atención Primaria de Salud: un Enfoque Fenomenológico
}

Marcela García Vera ${ }^{1}$, Miriam Aparecida Barbosa Merighi ${ }^{2}$, Claudete Aparecida Conz ${ }^{3}$, Marcelo Henrique da Silva ${ }^{4}$, Deíse Moura de Oliveira ${ }^{5}$, Maria Cristina Pinto de Jesus ${ }^{6}$, Luz Angélica Muñoz González ${ }^{7}$

\footnotetext{
${ }^{1}$ Enfermera, Doctora en Enfermería. Centro de Salud Familiar Corvallis. Antofagasta. Chile. E-mail: marcelagarciavera@gmail.com.

2 Enfermera, Doctora en Enfermería. Profesora Decana de la Escuela de Enfermería de la Universidad de São Paulo. São Paulo, SP, Brasil. E-mail: merighi@usp.br.

${ }^{3}$ Enfermera, Maestra en Enfermería. Alumna del Programa de Posgrado en Enfermería de la Escuela de Enfermería de la Universidad de São Paulo, nivel Doctorado. São Paulo, SP, Brasil. E-mail: clauenf@uol.com.br.

${ }^{4}$ Enfermero, Doctor en Enfermería. Enfermero de la Alcaldía Municipal de Juiz de Fora. Juiz de Fora, MG, Brasil. E-mail: marcelohenfermar@gmail.com.

${ }^{5}$ Enfermera, Doctora en Enfermería. Profesora Adjunta de la Universidad Federal de Viçosa. Viçosa, MG, Brasil. E-mail: dmouradeoliveira@gmail.com.

${ }^{6}$ Enfermera, Doctora en Enfermería. Profesora Decana de la Universidad Federal de Juiz de Fora. Juiz de Fora, MG, Brasil. Email: mariacristina.jesus@ufjf.edu.br.

${ }^{7}$ Enfermera. Profesora Decana de la Facultad de Enfermería, Universidad Andrés Bello. Santiago, Chile, E-mail: Imunoz@unab.cl.
}

Recebido: 21/05/2016.

Aceito: 22/05/2017.

Publicado: 02/10/2017.

\section{RESUMO}

Se buscó comprender el desempeño de las enfermeras chilenas en la Atención Primaria de Salud. Investigación cualitativa con enfoque desde la fenomenología social. La recolección de datos fue realizada entre enero y abril del 2013, a través de entrevista, con 13 enfermeras. El desempeño de las enfermeras muestra que ellas se ven con más autonomia profesional, mayor cercania a los usuarios, sin embargo, se sienten desgastadas en el trabajo. Sus expectativas incluyen el deseo de reconocimiento de su labor y una mejor gestión del servicio. Este estudio aporta como contribuciones la posibilidad de pensar nuevos diseños para las prácticas profesionales y de gestión en la Atención Primaria de Salud, desde la perspectiva de las enfermeras chilenas. Estos diseños se deben basar en la reorganización del proceso de trabajo y alcanzar los espacios de la macropolítica, influyendo el desempeño profesional de la enfermera en este nivel de atención de salud.

Descritores: Enfermería en Salud Comunitaria; Atención Primaria de Salud; Investigación Cualitativa.

\section{INTRODUCCIÓN}

Las reformas de los sistemas de salud se han producido a través de todo el mundo. Este cambio se fundamenta en la implementación de un modelo centrado en la Atención Primaria de Salud (APS), en el cual las enfermeras tienen una importante participación. Estudios

realizados en diferentes países ratifican esta afirmación ${ }^{(1-5)}$.

En Australia, resultados de un estudio que evaluó la práctica de las enfermeras y exploró las tendencias 
de su desempeño, indicó que las iniciativas de gobierno para fortalecer la atención básica, contribuirán en la última década al rápido crecimiento de la práctica de enfermería en ese nivel de atención ${ }^{(1)}$.

Una revisión de la literatura sobre las prácticas de enfermería, en el contexto de la Atención Primaria de Salud en Brasil, identificó prácticas en el ámbito de los servicios en la comunidad y aquellas relacionadas a la gestión y la formación. Hizo hincapié que hubo avances en la implantación de políticas de reorganización del trabajo en APS, sin embargo la enfermería brasileña necesita colocar la consulta ampliada como un imperativo ético-político de la organización de los servicios y de la intervención profesional ${ }^{(2)}$.

En Canadá, una revisión de la literatura mostró que fue posible la transformación de los cuidados en APS, a pesar que en el país convive un sistema pluralista de prestadores de salud privados. Para eso fue necesario un fuerte liderazgo gubernamental y profesional los que actuaron en conjunto en la búsqueda de mejorar la calidad del sistema ${ }^{(3)}$. Otro estudio canadiense identificó el impacto del desempeño de las enfermeras cuando ellas realizaban prácticas colaborativas con otros profesionales de APS. Se percibió mejoría en la calidad de la educación al paciente, enfocada hacia la promoción de la salud y prevención de enfermedades y con más tiempo para un examen detallado de la condición del paciente o una atención más completa $^{(4)}$.

Un estudio que tuvo como objetivo conocer cómo los países de América Latina, en especial Chile, incorporaron las normas y aspectos legales que genera el cuidado, destaca que el papel de las enfermeras debe estar bien definido por los gestores, tanto en el ámbito de la práctica como en el de la gestión, para que haya efectividad en su trabajo cotidiano y puedan evaluar su desempeño. Esto puede permitir el logro de la competencia y la autonomía profesional ${ }^{(5)}$.

En Chile, una investigación describe cómo las enfermeras perciben la implementación del Modelo de Atención Integral con Enfoque Familiar y Comunitario, ocurrido a partir de 1995. Mostró que una de las barreras para la aplicabilidad del servicio y el cumplimiento de las estrategias y normas de atención a la familia, es el énfasis en una política basada en procesos de salud, a expensas del respeto, la empatía y el compromiso de los profesionales sanitarios con la comunidad $^{(6)}$.

Se destaca que luego de la implementación de la APS en Chile, en la década de 1990, ha sido necesario que las enfermeras replanteen su desempeño frente a este modelo. Considerando que el trabajo de la APS en Chile, requiere de la redefinición de las prácticas de salud, conflictos y dificultades pueden surgir en el desempeño de sus actividades profesionales si no se produce una adecuación a este contexto específico.

La literatura señala contradicciones acerca del modo como la enfermera percibe su desempeño profesional frente a la autonomía conferida en la APS ${ }^{(7)}$. Eso demuestra la necesidad de más estudios sobre como este profesional se percibe actuando en este nivel de atención de salud.

Ante las consideraciones mencionadas surgen algunas preguntas: ¿cómo las enfermeras chilenas perciben su trabajo cotidiano en Atención Primaria de Salud?, ¿qué esperan en relación a su desempeño profesional? Tales preguntas culminaron en el objetivo del estudio: comprender el desempeño de las enfermeras chilenas que trabajan en Atención Primaria de Salud. 
La comprensión del fenómeno estudiado señalará aspectos de la vivencia de las enfermeras que trabajan en APS, llamando a las mismas a reflexionar sobre la práctica en el campo de la salud pública. Tal comprensión también puede apoyar la educación en el área de la APS, fomentar la educación continúa de estos profesionales en ese contexto, así como aumentar el conocimiento sobre el desempeño de la enfermera en la APS.

\section{MÉTODO}

Estudio cualitativo, basado en la fenomenología social de Alfred Schütz. Este referencial busca comprender las relaciones a partir de la acción de un determinado grupo social. Los presupuestos conceptuales como relación social, intersubjetividad, acervo de conocimientos, la motivación y acción social, se han utilizado para apoyar la discusión de los resultados ${ }^{(8)}$.

La acción del hombre en las relaciones sociales se orienta por su situación biográfica, que consiste en una historia sedimentada de experiencias subjetivas previas. Esa experiencia aporta una riqueza de conocimiento que está disponible y accesible de acuerdo con la situación biográfica. Este acervo está constituído por las informaciones transmitida a través de los padres, los educadores y por las experiencias vividas que estructuran este conjunto de conocimientos ${ }^{(8)}$.

La situación biográfica y el acervo de conocimientos disponibles y accesibles, fundamentan la proyección de la acción en el mundo social. Esta acción es interpretada por la persona a partir de motivos existenciales derivados de sus experiencias inscritas en la subjetividad, los que se constituyen en hilos conductores de la acción. Aquellos que se basan en la historia, en el acervo de conocimientos y la experiencia vivida en el ámbito biopsicosocial, son denominados "motivos porque" y los relacionados con el logro de objetivos, expectativas y proyectos son llamados "motivos para"(8).

La acción considerada en esta investigación está inscrita en un contexto de motivación, compuesto por las experiencias previas y actuales de las enfermeras que se desempeñan en APS (motivos porque) y por los proyectos que conducen a concretar la acción (motivos para).

El foco del estudio se centró en las enfermeras que se desempeñan en Atención Primaria de Salud, en donde trabajan un total de 45 enfermeras. Se solicitó como criterio de selección desempeñarse en APS.

La aproximación a los participantes fue en la reunión mensual de enfermeras. Se expuso la iniciativa y se ofreció la posibilidad de participar. Quienes manifestaron interés y cumplían con el criterio de selección fueron contactadas telefónicamente, acordando la entrevista en lugar y hora de su conveniencia.

No se determinó con antelación el número de participantes, ya que las entrevistas fueron completadas cuando se percibió que éstas eran suficientes para responder a los objetivos e inquietudes del estudio ${ }^{(9)}$. Entre los meses de enero y abril del 2013, fueron entrevistadas 13 enfermeras que accedieron a participar del estudio, cumplieron con los criterios de selección y firmaron el consentimento informado.

Las entrevistas fueron grabadas con el consentimiento de las enfermeras, tuvieron una duración promedio de 40 minutos y fueron conducidas y transcritas por la investigadora, a través de las siguientes 
preguntas orientadoras: ¿cómo es para usted actualmente, desempeñarse en APS?, ¿cuáles son sus expectativas en relación a su trabajo?. El material se clasificó, categorizó y analizó según los pasos propuestos por investigadores de la fenomenología social ${ }^{(10)}$.

El análisis de las entrevistas se dio a través de lectura y relectura atenta de las declaraciones, buscando siempre comprender la esencia de la vivencia de las enfermeras chilenas que se desempeñan en APSlos aspectos significativosificativos extra chilenas que se desempeñan en APS; agrupando los aspectos significativos extraídos de las entrevistas por medio de las convergencias temáticas, agrupadas con el objetivo de aprehender el significado que las enfermeras atribuyen a sus propias acciones; análisis de esas categorías buscando la comprensión de los "motivos porque" y "motivos para" de la acción de las enfermeras en APS y la discusión de los resultados a la luz de la fenomenología social y otros referenciales sobre el objeto del estudio.

Las declaraciones se identificaron con la letra E (enfermera) seguidas de un número según las entrevistas realizadas (E1 a E13). El Comité de Ética de la Facultad de Enfermería de la Universidad Andrés Bello, aprobó el proyecto mediante protocolo L1/CECENF/71.

\section{RESULTADOS}

Del análisis comprensivo de las actividades ya realizadas en el cotidiano de la APS, surgen categorias que revelan la reflexión de las enfermeras sobre su trabajo (motivos porque): "Más autonomía en las acciones profesionales", "Relación con los usuarios" y "Desgaste en el trabajo". Las categorias relacionadas a los "motivos para", muestran las expectativas de las enfermeras que se desempeñan en APS: "Espera reconocimiento por su trabajo" y "Espera mejor gestión en la APS".

\section{Categoria 1: Más autonomía en las acciones profesionales}

De acuerdo con las declaraciones, en APS, las enfermeras realizan sus actividades com mayor autonomía:

[...] me gusta trabajar en APS, porque aquí me considero uma persona autónoma, que no depende de otros para tener que tomar alguna resolución. (E5)

Tienes más independencia, nunca hemos estado supeditadas a los médicos, compartimos decisiones [...] es la decisión del médico y de la enfermera y en conjunto cuando se requiera, eso me gusta de APS. (E10)

[...] es el mejor lugar donde se puede trabajar [...] mayor capacidad de desarrollar la autonomía de la profesión, aquí en APS puede planificar y resolver problemas. (E11)

\section{Categoria 2: Relación con los usuarios}

Las enfermeras consideran que desempeñándose en APS tienen más oportunidades de desarrollar una relación con los usuarios:

Es gratificante, porque me gusta el contacto con la gente. No me imagino en un hospital, porque es más impersonal. [...] me gusta APS, no lo cambiaría. (E2) 
[...] si tienen situaciones con la familia, siempre me cuentan porque llevo tanto tiempo con ellos que hay una relación de cercania, hay más confianza. (E7)

Ellos se dan cuenta si eres cercana, si lo que dices lo haces con buena intención y en base a eso tienes más credibilidad. (E4)

\section{Categoria 3: Desgaste en el trabajo}

Las declaraciones de las enfermeras que se desempeñan en APS, muestran desgaste en el trabajo, desmotivándolas:

[...] tú sientes que a veces se te acaban las fuerzas, como que no tienes todos los medios que necesitas para hacer un montón de cosas, pero es el día a día, es necesaria la automotivación. (E9)

[...] te cargan y cargan más cosas, eso me tiene frustrada y un poco desmotivada. (E6) [...] tengo 15 pacientes con curaciones de pie diabético y vasculares y trabajo mucho. Toda la tarde la dejo para curaciones y para pasar en el computador el registro de las cartolas de los pacientes crónicos, eso es a diario. (E13)

\section{Categoria 4: Espera reconocimiento de su trabajo}

La enfermera expresa el deseo de tener su trabajo en APS, valorizado y reconocido porque se siente frustrada y muchas veces, invisible en el equipo de salud:

[...] ya no me siento importante dentro del equipo, pienso que la enfermera está siendo cada vez más invisible [...] me siento invisible. (E12)

Somos presionadas y no vemos reconocimiento, no espero grandes cosas, pero saber que las personas no reconocen tu trabajo, eso es lo que me da pena [...] y no es que sólo yo lo sienta, lo sentimos todas igual. (E3)

[...] de repente siento que la frustación parte de mí obviamente. [...] creo que tengo buenas ideas, pero siento que choco contra una pared, no me siento reconocida en lo que hago. (E6)

\section{Categoria 5: Espera mejor gestión en la APS}

El deseo de mejorar la gestión de la APS y la esperanza de poder contar con liderazgos competentes están expresados en las declaraciones:

[...] veo que acá en este nivel de atención, hay gente que tiene mucho potencial, pero por la estructura que tiene el sistema, no se aprovecha (E1).

[...] mis expectativas son cambiar la gestión de algunas áreas para que se hagan las cosas mejor. La gestión es mala en cuanto a la dirección; quienes están en segundo plano toman las decisiones [...] a veces no son las mejores [...]. (E4) No siempre estás respaldada por tu jefe... acá nadie quiere asumir más responsabilidades respecto a estar liderando un grupo de trabajo [...] no hay apoyo al profesional. (E10)

\section{DISCUSIÓN}

Con respecto al desempeño profesional en la APS, las enfermeras del presente estudio destacan que este es el escenario de atención de salud donde poseen mayor autonomía. Estudios refieren que la autonomía deseada por esta profesional constituye un factor que se debe considerar al momento de evaluar su desempeño laboral, como una de las ventajas que ofrece ese lugar de trabajo. Esto se debe principalmente, a que las intervenciones gozan de mayor independencia en comparación con otros ámbitos 
del sector salud, permitiéndoles un cuidado más autónomo ${ }^{(11)}$.

Una revisión integrativa de la literatura mostró que gran parte de los estudios destacan que la autonomía de la enfermera en APS, aún no está plenamente consolidada, debido al perfil profesional y las condiciones adversas del proceso laboral. No obstante, señalan que algunos estudios mostraron que esta autonomía es real y resulta del esfuerzo conjunto de los trabajadores de enfermería ${ }^{(7)}$.

En Portugal, una investigación analisó el trabajo del equipo en APS, mostrando que uno de los factores que contribuía a una mayor motivación y satisfacción en el trabajo era la autonomia en temas técnicooperacionales, que conducía a mejorar la capacidad de resolución de los problemas de salud encontrados ${ }^{(12)}$.

En la ciudad de Ontario, un estudio realizado con enfermeras canadienses, señaló que la autonomía de las enfermeras de APS, se desarrolla en la capacidad de ejercer su práctica, tomar decisiones y dirigir la gestión de los cuidados de salud. Eso genera niveles más altos de satisfacción en el trabajo ${ }^{(13)}$.

Otro tema destacado por las enfermeras chilenas acerca de su labor en APS, tiene que ver con la posibilidad de establecer una relación de mayor cercania con los usuarios.

Un estudio de revisión de la literatura mostró que en el aspecto técnico-asistencial la APS de Brasil, reorientada por la Estrategia de Salud de la Familia, ha alcanzado mejor desempeño que el modelo de APS tradicional. Eso se debe al trabajo interdisciplinar con enfoque familiar que valoriza el acogimiento, el vínculo y la orientación comunitaria ${ }^{(14)}$.

De acuerdo con la premisa de que la acogida proporciona la creación de un vínculo con la comunidad, los resultados de estudio realizado con usuarios del Sistema Único de Salud (SUS) brasileño en Belo Horizonte, indicaron que éstos perciben a la enfermera como el profesional de salud capaz de realizar un abordaje acogedor que proporciona cercanía y valora el diálogo y que con ella consigue expresar más facilmente sus necesidades de salud. Los usuarios consideran que esta actitud de la enfermera facilita el establecimiento de un vínculo de confianza con el profesional ${ }^{(15)}$.

Así, el trabajo de la enfermera más que una actividad, es una forma de relación social denominada "cara a cara" y considerada por Schutz, el tipo más auténtico de las relaciones humanas ${ }^{(8)}$. Es intersubjetiva porque los seres humanos se vinculan, comprendiendo el sentido involucrado en las relaciones sociales ${ }^{(8)}$.

Aunque las enfermeras se perciben con mayor autonomía y mencionan centrarse en una relación de intersubjetividad con los usuarios del sistema de salud, enfatizan el desgaste profesional causado por la sobrecarga de trabajo.

Un estudio reveló factores favorables y desfavorables relativos a la ampliación de las funciones de la enfermera del Servicio Nacional de Salud de Inglaterra, en la APS. Señaló un mayor reconocimiento profesional por parte de los pacientes y demás profesionales, aumento del poder técnico y del cuerpo de conocimientos profesionales, además de una mayor satisfacción en el trabajo, en función del aumento de la resolutividad de su práctica. Por otro lado, mostró la sobrecarga de trabajo ya que la enfermera pasó a agregar a las actividades cotidianas, las prácticas adicionales ${ }^{(16)}$.

También en Chile, una investigación realizada con las enfermeras de APS, mostró que en la fase de 
consolidación de la implementación del modelo de salud familiar surgió el desgaste por estrés laboral, que generaba ausentismo laboral y rotación de profesionales ${ }^{(6)}$.

El acervo de conocimientos de las enfermeras ${ }^{(8)}$, retratado en las categorías anteriores lleva a estas profesionales a reflexionar sobre el contexto de la APS y a expresar expectativas sobre su desempeño en ese nivel de atención (motivos para).

La necesidad de reconocimiento que las enfermeras de APS, perciben por parte de los profesionales, gestores y de la comunidad y es demostrada en este estudio por medio de los relatos, muestran que la valoración y el reconocimiento, facilitan brindar cuidados contribuyendo a la realización personal y profesional.

Un estudio realizado con enfermeras españolas mostró que el reconocimiento de enfermería está relacionado con la valoración del cuidado y que tanto la tecnología como la burocracia involucrada en los cuidados, reducen el tiempo invertido junto al paciente. En ese sentido cuando el cuidado se vuelve invisible, la autonomía y el reconocimiento de la profesión están amenazadas ${ }^{(17)}$.

Otro estudio realizado con las enfermeras que trabajan en APS, en Brasil, mostró que la mayor visibilidad social es a través de la ejecución de la variedad de cuidados que ellas ofrecen y los diversos ámbitos de trabajo. Las enfermeras están en constante búsqueda de lo nuevo, con la realización de prácticas que tienen como objetivo la integralidad de la asistencia y el compromiso con la resolutividad de los problemas y necesidades de la población ${ }^{(18)}$.

Las enfermeras chilenas que participaron del presente estudio no solamente esperan reconocimiento, sino también esperan una mejor gestión de la APS. Del mismo modo una investigación realizada en Escocia, con enfermeras comunitarias, mostró que ellas esperan que la gestión elimine prácticas burocráticas que no contribuyen al trabajo cotidiano, desean líderes con probadas capacidades gerenciales que conozcan el ámbito de APS, cercanos y proclives a generar espacios de participación, es decir, un liderazgo del tipo transformacional ${ }^{(19)}$.

Coincide con los resultados del presente estudio, los hallazgos de investigaciones con enfermeras brasileñas que trabajan en APS. Se destaca en ellos que los factores que contribuyen a la satisfacción laboral se relacionan a una política gerencial más organizada en las instituciones públicas, continuar mejorando el sistema de salud y mejor definición de sus funciones por los gestores ${ }^{(20)}$.

Se observa que los procesos de cambio necesarios en la APS, ocurren paulatinamente, siendo imprescindible invertir en diversas áreas, especialmente en el desarrollo de la gestión, de la formación y educación permanente en salud, en el fortalecimiento del control social, en la evaluación participativa en el sistema de salud y en la mejora de tecnologías para la gestión y el cuidado ${ }^{(21)}$.

\section{CONCLUSIÓN}

El desempeño profesional de las enfermeras en APS, a partir de la fenomendologia social de Alfred Schütz, se mostró a través del desempeño de modo autónomo, en una relación de proximidad con el usuario, 
sin embargo, desgastante. Estas profesionales esperan reconocimiento por parte del equipo, gestores y comunidade, liderazgos competentes y sin sobrecarga de actividades.

Como limitación de este estudio, se destaca que como en toda investigación cualitativa el fenómeno nunca es plenamente develado, por lo que es perspectival. Los resultados se generan y aplican a este grupo social específico, que se desarrolla en una realidad local concreta, con características geográficas, culturales y administrativas particulares. Por esta razón, nuevos estudios pueden complementar este conocimiento y contrastar el desempeño de la enfermera en APS, en otras realidades.

Este estudio ha traído como contribución la posibilidad de idear nuevos diseños en las prácticas profesionales y de gestión en la Atención Primaria de Salud, desde la perspectiva de las enfermeras chilenas. Estos diseños deben alcanzar los espacios de la macropolítica y estar basados en la reorganización de los procesos de trabajo, influyendo en el desempeño profesional de la enfermera en este nivel de atención de salud.

\section{REFERENCIAS}

1. Halcomb EJ, Salamonson Y, Davidson PM, Kaur R, Young SA. The evolution of nursing in Australian general practice: a comparative analysis of workforce surveys ten years on. BMC Fam Pract [Internet]. 2014 [citado 2017 Oct 01];15:52. Disponible en: http://dx.doi.org/10.1186/1471-2296-15-52.

2. Barbiani R, Nora CRD, Schaefer R. Nursing practices in the primary health care context: a scoping review. Rev Lat Am Enfermagem [Internet]. 2016 [citado 2017 Oct 01];24:e2721. Disponible en

: http://dx.doi.org/10.1590/1518-8345.0880.2721.

3. Hutchison B, Levesque JF, Strumpf E, Coyle N. Primary health care in Canada: systems in motion. Milbank Q [Internet]. 2011 [citado 2017 Oct 01];89(2):256-88. Disponible en: http://dx.doi.org/10.1111/j.14680009.2011.00628.x.

4. Roots A, MacDonald M. Outcomes associated with nurse practitioners in collaborative practice with general practitioners in rural settings in Canada: a mixed methods study. Hum Resour Health [Internet]. 2014 [citado 2017 Oct 01];12:69. Disponible en: http://dx.doi.org/10.1186/1478-4491-12-69.

5. Milos Hurtado P, Bórquez Polloni B, Larrain Sundt AI. La "gestión del cuidado" en la legislación chilena (II) estado actual. Cienc y enfermería [Internet]. 2011 [citado 2017 Oct 01];17(3):23-33. Disponible en:

http://dx.doi.org/10.4067/S0717-95532011000300003.

6. Cárdenas $\mathrm{C}$, Pinninghoff $\mathrm{C}$. Modelo integral de salud con enfoque familiar y comunitario: experiencias en la implementación desde un equipo de atención hospitalaria. Rev Chil Salud Pública [Internet]. 2013 [citado 2017 Oct 01];17(2):139-46. Disponible en: http://www.revistasaludpublica.uchile.cl/index.php/RCSP/article/view/27114/28745. 7. Chaves ACC, Medeiros SM, Oliveira JSA, Gomes MGCGP. Autonomia profissional do enfermeiro na estratégia saúde da família: revisão integrativa. Revista de enfermagem UFPE on line [Internet]. 2014 [citado 2017 Oct 01];8(supl. 2):3718-26. Disponible en: http://www.revista.ufpe.br/revistaenfermagem/index.php/revista/article/view/4812 8. Schütz A. El problema de la realidad social, Escritos I. Buenos Aires: Amorrortu. 2008.

9. Martínez-Salgado C. El muestreo en investigación cualitativa: principios básicos y algunas controversias. Cien Saude Colet [Internet]. 2012 [citado 2017 Oct 01];17(3):613-9. Disponible en: http://dx.doi.org/10.1590/S141381232012000300006.

10. Jesus MCP, Capalbo C, Merighi MAB, Oliveira DM, Tocantins FR, Rodrigues BMRD, et al. A fenomenologia social de Alfred Schütz e sua contribuição para a enfermagem. Rev Esc Enferm USP [Internet]. 2013 [citado 2017 Oct 01];47(3):736-41. Disponible en Disponible en : http://dx.doi.org/10.1590/50080-623420130000300030. 
11. Matumoto S, Fortuna CM, Kawata LS, Mishima SM, Pereira MJB. Nurses' clinical practice in primary care: a process under construction. Rev Lat Am Enfermagem [Internet]. 2011 [citado 2017 Oct 01];19(1):123-30. Disponible en: http://dx.doi.org/10.1590/S0104-11692011000100017.

12. Souza MB, Rocha PM, Sá AB, Uchoa SAC. Trabalho em equipe na atenção primária: a experiência de Portugal. Rev Panam Salud Pública [Internet]. 2013 [citado 2017 Oct 01];33(3):190-5. Disponible en:

http://dx.doi.org/10.1590/S1020-49892013000300005.

13. Regan S, Laschinger HK, Wong CA. The influence of empowerment, authentic leadership, and professional practice environments on nurses' perceived interprofessional collaboration. J Nurs Manag [Internet]. 2016 [citado 2017 Oct 01];24(1):E54-61. Disponible en: http://dx.doi.org/10.1111/jonm.12288.

14. Arantes LJ, Shimizu HE, Merchán-Hamann E. Contribuições e desafios da Estratégia Saúde da Família na Atenção Primária à Saúde no Brasil: revisão da literatura. Cien Saude Colet [Internet]. 2016 [citado 2017 Oct 01];21(5):1499510. Disponible en: http://dx.doi.org/10.1590/1413-81232015215.19602015.

15. Souza PA, Batista RCR, Lisboa SF, Costa VB, Moreira LR. Percepção dos usuários da atenção básica acerca da consulta de enfermagem. REME Rev Min Enferm [Internet]. 2013 [citado 2017 Oct 01];17(1):11-7. Disponible en : http://www.dx.doi.org/10.5935/1415-2762.20130002.

16. Toso BRGO, Filippon J, Giovanella L. Atuação do enfermeiro na Atenção Primária no Serviço Nacional de Saúde da Inglaterra. Rev Bras Enferm [Internet]. 2016 [citado 2017 Oct 01];69(1):182-91. Disponible en:

http://dx.doi.org/10.1590/0034-7167.2016690124i.

17. Galbany-Estragués P, Comas-d'Argemir D. Care, Autonomy, and Gender in Nursing Practice: A Historical Study of Nurses' Experiences. J Nurs Res [Internet]. 2017 [citado 2017 Oct 01];25(5):361-7. Disponible en:

http://dx.doi.org/10.1097/jnr.0000000000000184.

18. Viegas SMF, Penna CMM. Práticas integrais na estratégia saúde da família no Brasil: o quotidiano do trabalho em equipa. Rev Enferm Ref [Internet]. 2013 [citado 2017 Oct 01];serllI(10):99-108. Disponible en:

http://www.scielo.mec.pt/scielo.php?script=sci_arttext\&pid=S0874-02832013000200012.

19. Cameron S, Harbison J, Lambert V, Dickson C. Exploring leadership in community nursing teams. J Adv Nurs [Internet]. 2012 [citado 2017 Oct 01];68(7):1469-81. Disponible en: http://dx.doi.org/10.1111/j.1365-

2648.2011.05869.x.

20. Junqueira MAB, Santos FCS. A educação em saúde na Estratégia Saúde da Família sob a perspectiva do enfermeiro: uma revisão de literatura. Revista de Educação Popular[Internet]. 2013 [citado 2017 Oct 01];12(1):66-80. Disponible en: http://www.seer.ufu.br/index.php/reveducpop/article/view/20301.

21. Santana FR, Santana FR, Anjos GV, Campos TV, Lima PCT, Lopes MM, et al. Ações de saúde na estratégia saúde da família no município goiano na perspectiva da integralidade. Rev. Eletr. Enf. [Internet]. 2013 [citado 2017 Oct 01];15(2):422-9. Disponible en: http://dx.doi.org/10.5216/ree.v15i2.16936. 